\section{The effect of the discounted attribute importance in two-sided messages}

Discounted attribute importance

\author{
Cristiane Pizzutti
}

School of Management, Universidade Federal do Rio Grande do Sul, Porto Alegre, Brazil

Kenny Basso

IMED Business School, Faculdade Meridional, Passo Fundo, Brazil, and

Manuela Gonçalves

Business School, Universidade Feevale, Novo Hamburgo, Brazil

\begin{abstract}
Purpose - The purpose of this research is to test the importance of the discounting attribute in the two-sided communication from a retail salesperson as a boundary condition that eliminates the trade-off between trustworthiness and purchase intentions.

Design/methodology/approach - The hypotheses are tested by three experimental studies in three different retail contexts. Two lab studies manipulate the importance of the attribute and the type of message: one-sided vs two-sided. A field study improves the external validity of the findings.

Findings - A two-sided message from a salesperson reduces the use of persuasion knowledge and, therefore, enhances the consumer's perception of the salesperson's trustworthiness; this positive effect remains significant across different levels of importance of the discounting attribute. A two-sided message decreases the consumer's probability of purchase only when an important attribute is disclaimed, through the consumer's beliefs regarding the product's attributes.

Practical implications - For the appropriate use of two-sided appeals, retailers should identify the importance of product attributes from the consumers' perspective. A negative remark from a salesperson when referred to an unimportant attribute makes no harm to purchase intentions while leading to stronger intentions to return to the store and to recommend the store by enhancing trustworthiness.

Originality/value - This paper shows that it is possible to enhance trustworthiness through a two-sided message without mitigating the intentions of buying by discounting an attribute at low importance in the two-sided message.
\end{abstract}

Keywords Knowledge, Persuasion, Trustworthiness, Purchase attribute importance,

Two-sided message

Paper type Research paper

\section{Introduction}

Imagine you are in a store and take a shirt off the rack. At this moment, the salesperson comes and says, "This shirt has a good fit and is very comfortable". What would you think? Is the salesperson trying to persuade you? However, if she had said, "This shirt has a good fit but needs to be ironed", would you feel the same? Vol. 50 No. $9 / 10,2016$ p. 000 0309-0566 DOI 10.1108/EJM-05-2015-0304 
EJM

$50,9 / 10$

Previous research suggests that consumers use their persuasion knowledge and consider a salesperson's advice given prior to a sale as an attempt to encourage a purchase and thus discount the advice (Campbell and Kirmani, 2000; Friestad and Wright, 1994, 1999; Shu and Carlson, 2014). However, the two-sided communication literature (Crowley and Hoyer, 1994; Golden and Alpert, 1987; Kamins et al., 1989) indicates that consumers might sometimes regard advice given prior to a sale as sincere. The main empirical finding from this literature indicates that a two-sided message -i.e. a message that communicates both positive and negative attributes of a product enhances source trustworthiness (Eisend, 2007; Golden and Alpert, 1987; Kamins et al., 1989; Smith and Hunt, 1978) because the inclusion of negative information is not expected and leads the recipient of the message to conclude that the source is speaking truthfully.

Studies also indicate that a trade-off may occur when two-sided communication is employed. Although a negative comment leads to higher consumer perception of the source's trustworthiness, the revelation that an attribute of the product is undesirable may lead to a lower intention to purchase (Pechmann, 1992; Eisend, 2007).

Nevertheless, empirical studies have produced mixed results regarding the impact of two-sided messages on purchase intentions. The results from these studies have been either nonsignificant or mixed (Kamins and Marks, 1987; Sawyer, 1973; Stayman et al., 1987; Swinyard, 1981) or favored the two-sided treatment (Etgar and Goodwin, 1982; Golden and Alpert, 1987; Kamins, 1989; Kamins et al., 1989).

These contradictory findings can be attributed, at least in part, to differences among the various studies in the level of importance of the attributes disclaimed. However, despite the calls for more research on this topic (Crowley and Hoyer, 1994), little research has systematically manipulated the importance of the disclaimed attribute to investigate its impact on communication effectiveness (exceptions are Stayman et al., 1987; Okechuku et al., 1993).

This paper seeks to answer the following question:

Q1. Is the two-sided communication trade-off between a salesperson's trustworthiness and a consumer's purchase intentions unavoidable or might the trade-off be conditional on the importance of the discounting information?

We propose the importance of the discounting attribute in two-sided communication as a boundary condition that may reduce or even eliminate the trade-off. When the discounting attribute is important, there will be a trade-off between consumers' perceptions of the salesperson's trustworthiness (enhanced) and their purchase intentions (decreased). However, when the discounting attribute is unimportant, the trade-off is mitigated, i.e. trustworthiness increases, but purchase intentions do not decrease as sharply as with a one-sided message.

The findings of our study make several contributions. First, contributing to the two-sided message literature, our study sheds new light on the critical role of the importance of the discounted attribute and the effect of its interaction with the sidedness message on trustworthiness and purchase intentions, showing that:

- contrary to what is proposed in the extant literature, a two-sided message leads to stronger perceptions of sales agent trustworthiness than a one-sided message, regardless of the level of importance of the attributes linked to the remarks; and 
- a two-sided message does not reduce purchase intentions when an unimportant attribute is discounted.

Discounted attribute importance

However, although it is possible in this case to not decrease the purchase intention after a two-sided message, the level of intention is not higher than the one achieved by a one-sided message. Second, our findings highlight the mechanism through which the effects on trustworthiness occur: persuasion knowledge. Third, we found an indirect effect of the interaction between the sidedness message and the importance of the discounted attribute on purchase intentions and on other behavioral intentions (i.e. recommend the store and return to the store) through salesperson trustworthiness.

\section{The trade-off of two-sided messages in the retail setting}

Gains in trustworthiness - the effects of two-sided messages on trustworthiness

Two-sided communication provides information about both the positive and negative attributes of a product or service (Crowley and Hoyer, 1994). Researchers advocate that including negative information about a product may be more effective than presenting only positive information (Cornelis et al., 2014; Ein-Gar et al., 2012; Eisend, 2006, 2010; Etgar and Goodwin, 1982; Golden and Alpert, 1987; Kamins and Assael, 1987; Pechmann, 1992). In fact, empirical findings consistently indicate that two-sided advertising can enhance trustworthiness (Golden and Alpert, 1987; Kamins and Marks, 1987; Smith and Hunt, 1978).

The literature shows that trustworthiness and expertise are the two most important components of credibility (Eagly and Chaiken, 1993; Hovland et al., 1953; Ohanian, 1990;

AQ: $1 \quad$ McGinnies and Ward, 1980). The effectiveness of two-sided advertising has been largely attributed to fostering the belief in the advertiser's willingness (rather than ability) to tell the truth (Schlosser, 2011). Following this logic, this paper focuses on trustworthiness.

Persuasion knowledge is a wisdom consumers develop about the tactics used in persuasion attempts to identify how, when and why marketers try to influence them (Friestad and Wright, 1994). Negative salesperson communications can affect consumers' inferences about persuasion motives underlying salesperson behavior and influence perceptions of salesperson trustworthiness as a consequence. Based on the extra credit effect, when a person makes negative evaluations to another contrary to what was expected, the evaluator may gain extra credit from the target. Hence, the target may form a better judgment of an evaluator who appears to have rejected the use of persuasion tactics (e.g. they have resisted the temptation to flatter) (Drachman et al., 1978).

The basis of the extra credit effect relies on the tenets of correspondence theory developed by Jones and Davis (1965). According to this theory, observers make a correspondent attribution for the cause of an event when they attribute an event's cause to the true feelings or disposition of the corresponding individual. Alternatively, observers make a non-correspondent attribution when they believe the cause of an event reflects situational constraints or pressures upon the individual (e.g. attempting to earn commissions).

Therefore, because the normal behavior that consumers expect from salespeople includes attempts to sell their products by communicating its superior features, consumers generally consider negative communications to be unusual (Smith and Hunt, 1978). Therefore, negative feedback from a salesperson should trigger a correspondent 
EJM

50,9/10

attribution and lead the consumer to assign the salesperson a higher degree of trustworthiness.

However, does the nature of the negative information have any impact on the positive effect a two-sided message has on trustworthiness? Intuitively, researchers have recognized that the perceived importance of the discounting attribute has a pivotal role on the effectiveness of two-sided advertising (Ein-Gar et al., 2012; Crowley and Hoyer, 1994; Kamins and Marks, 1987; Pechmann, 1992; Settle and Golden, 1974; Smith and Hunt, 1978). As a result, researchers (Etgar and Goodwin, 1982; Golden and Alpert, 1987; Hastak and Park, 1990) have simply disclaimed unimportant attributes in their studies, based on pre-tests. However, one could argue that, if the disclaimer is made on an unimportant attribute, it might not suffice to enhance trustworthiness. In this line, Stayman et al. (1987) explored the moderating role of the importance of the attributes in the effectiveness of two-sided messages and found that a two-sided message with an unimportant attribute was not perceived to be more trustworthy than a one-sided message. Furthermore, Okechuku et al. (1993) found results contrary to Stayman et al. (1987): when unimportant or moderately important attributes were disclaimed, trustworthiness was not significantly affected, whereas when highly important attributes were disclaimed, trustworthiness significantly decreased. Importantly, both authors did not present the mechanism that explains their results. Finally, other researchers have found that using discounting attributes of little importance in a two-sided message can be sufficient to enhance trustworthiness (Etgar and Goodwin, 1982; Golden and Alpert, 1987; Pechmann, 1992; Settle and Golden, 1974; Smith and Hunt, 1978), and Eisend (2006) found in his meta-analysis no sign of moderation of the importance of negative information.

As we can see, the effect of the level of the discounting attribute importance on trustworthiness is not a matter settled yet. However, once we propose that the effect of the message sidedness on trustworthiness is explained by the correspondence theory, it is likely that discounting more important attributes may lead to more correspondent attributions and, thus, to stronger trustworthiness perceptions. That is, if a relatively important attribute is discounted, the viewer may conclude that only a very honest source would make such a claim. Therefore, as the importance of the negative attribute is increased, trustworthiness is also increased (Crowley and Hoyer, 1994).

In sum, we propose that a two-sided message will lead to stronger perceptions of sales agent trustworthiness than a one-sided message, and, the higher the importance of the discounted attribute, the greater the trustworthiness associated to the salesperson.

In addition, consistent with calls for more research on the cognitive mechanisms consumers use to address persuasion (Ahluwalia, 2000; Bushman and Bonacci, 2002), we posit that activating persuasion knowledge will mediate the effect of the interaction between the message sidedness and the importance of the discounting attribute on consumer's perceptions of the salesperson trustworthiness.

This logic leads to the following hypotheses:

H1. A two-sided message from a salesperson (as opposed to a one-sided message) leads to a stronger increase on consumer's perceptions of the salesperson trustworthiness when the discounting attribute is important (as opposed to unimportant). 
H2. The effect of the interaction between the message sidedness (two-sided vs.one-sided) and the importance of the discounting attribute on consumer's perceptions of the salesperson trustworthiness is mediated by the activation of persuasion knowledge.

Discounted attribute importance

Losses in behavior - the effects of two-sided messages on purchase intentions

The cost of these gains in trustworthiness is the fact that sales agents must communicate negative information about their firm's products or services and risk losing the sale. On this regard, Settle and Golden (1974) found that losses in total expected benefits from the product offset any trustworthiness gains achieved by including negative claims.

Empirical studies have produced mixed results regarding the impact of two-sided messages on purchase intentions. These contradictory findings on the trade-off phenomenon indicate that it may be mediated by the characteristics of the negative information (Crowley and Hoyer, 1994). However, although some message characteristics have received research attention (e.g. the amount of negative information and correlations between positive and negative attributes), surprisingly little empirical research has systematically manipulated the importance of the disclaimed attribute to investigate its impact on communication effectiveness.

In the scenario in which a two-sided advertisement stating that a record store performed poorly on price - which is an important attribute - Stayman et al. (1987) found that respondents were less favorably disposed toward the store than those exposed to a one-sided ad. Conversely, when respondents were exposed to an unimportant attribute or to a moderately important attribute, they were no less favorably disposed toward the store than those exposed to the one-sided ad.

Consistent with these results, we propose that consumers evaluate the entire product based on the sum of its attributes. When a salesperson conveys a two-sided message disclaiming an important attribute, the discount that consumers give is intense enough to strongly affect their willingness to purchase the product. However, when the negative attribute is unimportant, the discount in their intention of purchasing may not be so intense. Therefore, disclaiming an attribute will reduce the probability of purchase, compared with one-sided communication, but the reduction will be stronger when the attribute is important.

Following this reasoning, we present $H 3$ :

H3. A two-sided message from a salesperson (as opposed to a one-sided message) leads to a stronger decrease on consumers' purchase intentions when the discounting attribute is important (as opposed to unimportant).

In addition, the literature is consistent regarding the relationship between trust feelings and purchase intentions (Sirdeshmuck et al., 2002; Swan et al., 1999). Swan et al. (1999), for instance, found that the perceived trustworthiness of a salesperson has a positive impact on clients' purchase intentions. The findings of DeCarlo (2005) support a link between a consumer's attitude toward the salesperson (e.g. is the salesperson truly motivated to help or motivated to make his commission?) and purchase intentions. On the effect of sidedness messages, Basso et al. (2014) show that delivering negative along with positive remarks (vs only positive remarks) enhances future patronage intentions through an increase of salesperson trustworthiness. Therefore, we propose that besides 
$\mathrm{EJM}$

$50,9 / 10$

6 the direct effect on purchase intentions, the sidedness message will have an indirect effect on purchase intentions through salesperson trustworthiness perceptions:

H4. The effect of the message sidedness (two-sided vs one-sided) on consumer's purchase intentions is mediated by the salesperson's trustworthiness.

Figure 1 presents a model of our conceptual propositions.

\section{Study 1}

We designed this study to initially test all hypotheses.

\section{Participants and design}

Participants consisted of 472 volunteers (56.5.9 per cent male, $M_{\text {age }}=33.59, S D=10.78$ ) who participated in an online survey by MTurk with the incentive of $\$ 0.50$. The only screening procedure used was the time completing the survey (too little or too much time, such that the respondent appeared to have started and then left off to do something else). None participant was removed from the analysis based on this requirement.

We used a 2(communication conditions: one-sided/two-sided) $\times 2$ (attribute conditions: important/non-important) between-subjects design, with random assignment.

\section{Procedures and stimuli}

Participants read a half-page scenario based on Campbell and Kirmani (2000). In the scenario, we asked participants to imagine they had gone to a store to look for a pair of jeans. The participant asks to try on the jeans. During this process, the salesperson makes a comment. To manipulate the communication condition, we inserted the salesperson's positive (e.g. these jeans have a good fit) or negative remark (e.g. these jeans need ironing) about the product.

We manipulated the importance of the attribute through two attributes: comfort and the need to be ironed. To find these two attributes, we performed a pretest with 61 subjects. The pretest indicated that fit $(M=6.30)$ and comfort $(M=6.25)$ were the two most important attributes from our list, with no difference between them $(p>0.05)$. Not needing ironing is not considered particularly important $(M=3.79)$ and is significantly less important than comfort and fit $(p<0.01)$. Based on the foregoing, we chose to use fit as a fixed attribute and comfort and the need to be ironed as manipulations of important and non-important attributes, respectively. Table I presents the four scenarios.

Figure 1.

Conceptual model

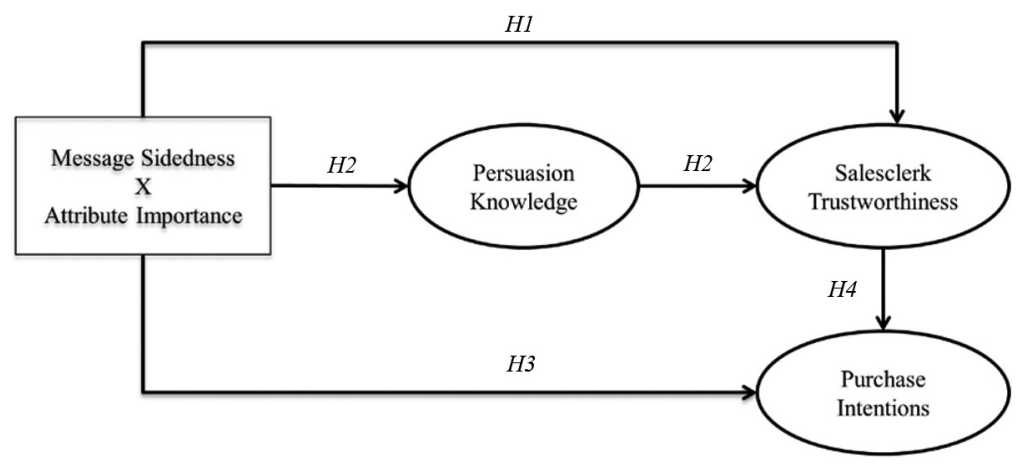


After reading the scenario, respondents answered items related to the dependent variables.

Discounted attribute importance

\section{Measures}

We adapted the trustworthiness index used in Main et al., (2007) using three seven-point Likert items (sincere/honest/trustworthy) $(\alpha=0.94)$. We measured the participant's purchase intentions using the following item (how likely would you be to purchase these jeans: very unlikely to very likely) on a seven-point scale. Neither gender nor age had an impact on participants' perceptions of the remark conditions or on the dependent variables $(p>0.10)$ in this or in any other experiment and will therefore not be discussed further.

Persuasion knowledge was measured through an open-ended question asking all the thoughts that participants had while reading a scenario. We identify 472 thoughts related to persuasion (each participant was responsible for one thought). We did not code general thoughts that were unrelated to persuasion. To determine the extent to which participants evoke attributional processes, two trained doctoral students coded these responses into one of the two following variables of attributional thoughts about sales agent behavior: either no evidence of ulterior motives linked to correspondent attribution, for example, the salesperson was honest or trying to help me (208 out of 472 thoughts -44.1 per cent); or evidence of ulterior motives linked to non-correspondent attribution, for example, the salesperson was trying to persuade me to make the purchase (264 out of 472 thoughts -55.9 per cent). We formulated the persuasion knowledge index as a dummy variable $(0=$ no evidence of ulterior motive and $1=$ evidence of ulterior motive). The agreement rate between the coders was 85.3 per cent, and the authors consensually resolved any differences.

We conducted a communication sidedness manipulation check using one question at the end of the questionnaire (the salesperson made positive and negative remarks while you were trying the jeans on - totally disagree/totally agree). To check the importance of the attributes, we asked the participants how important each attribute is for buying a pair of jeans (seven-point importance scale).

Supporting the validity of our scenario, the perceived realism (measured by a seven-point Likert item: "I can imagine myself in a similar situation") was higher ( $M=$ $5.74, p<0.001$ ) than the scale mid-point (4 on a scale of 1 to 7 ) in all experimental conditions. The same results were found in the next studies and will therefore not be discussed further.

Because the perceived correlation between the attributes may influence the results two-sided messages are more effective when the undesirable attribute is highly correlated with the desirable attribute featured in the message (Etgar and Goodwin

\begin{tabular}{|c|c|c|c|}
\hline \multicolumn{3}{|c|}{ Communication manipulation } & \\
\hline & One-sided & Two-sided & \\
\hline \multicolumn{4}{|c|}{ Attribute importance } \\
\hline Important & $\begin{array}{l}\text { These jeans have a good fit and are } \\
\text { comfortable as well }\end{array}$ & $\begin{array}{l}\text { These jeans have a good fit but are } \\
\text { not comfortable }\end{array}$ & Table I. \\
\hline Unimportant & $\begin{array}{l}\text { These jeans have a good fit and } \\
\text { also they do not need ironing }\end{array}$ & $\begin{array}{l}\text { These jeans have a good fit but } \\
\text { need ironing }\end{array}$ & $\begin{array}{r}\text { Scenarios used in } \\
\text { Study } 1\end{array}$ \\
\hline
\end{tabular}


EJM

$50,9 / 10$

1982; Pechmann, 1992) - we measured the extent to which the attributes were perceived as correlated. We followed Pechmann (1992) and used a nine-point scale ( -1 negatively correlated; 0 not correlated at all; +1 positively correlated). We found that there was a positive correlation perceived between fit and comfort $(M=0.47)$ and between fit and the need to be ironed ( $M=0.03)$; however, both correlations were moderate, which indicated that the participants did not perceive the attributes as interdependent.

\section{Results}

Manipulations checks. Participants in the two-sided condition correctly indicated a higher mean for the "salesperson made positive and negative remarks about the jeans" question $(M=5.63)$ than participants in the one-sided condition $(M=2.45 ; F[1,468]=$ $453.12, p<0.001)$. There is no effect of attribute manipulation $(F[1,468]=1.242, p=$ $0.266)$ or interaction $(F[1,468]=0.238, p=0.62)$ on the communication manipulation check.

We also verified whether the respondents perceived the importance of the attributes for choosing the jeans differently. In the condition of two important attributes, fit $(M=$ $5.98)$ and comfort $(M=5.99)$ do not present a significant difference $(t=-0.15$; $\mathrm{df}=251$; $p=0.87)$, whereas in the condition with an important and a non-important attribute, fit $(M=6.35)$ is more important than the necessity of ironing $(M=3.26 ; t=20.93$; $\mathrm{df}=219$; $p<0.001)$, and comfort $(M=5.99)$ is perceived as significantly more important than the necessity of ironing $(M=3.26 ; F[1,468]=403.24, p<0.001)$.

Neither message manipulation $(F[1,468]=2.565, p=0.11)$ nor interaction $(F[1,468]=$ $1.036, p=0.30)$ presented a significant effect on the attribute manipulation check.

Trustworthiness. The ANOVA model shows that attribute manipulation $(F[1,468]=$ $2.357, p=0.125)$ does not present a main effect, whereas the communication condition $(F$ $[1,468]=64.572, p<0.001)$ presents a significant main effect on salesperson trustworthiness. There is no interaction effect between attribute and communication manipulations on trustworthiness $(F[1,468]=0.000, p=0.98)$. As expected, by paired comparisons, when the salesperson makes only positive remarks (one-sided) $(M=4.52)$, perceived trustworthiness is lower than when the salesperson makes a two-sided communication $(M=5.40)$. However, trustworthiness is not significantly different $(p>$ 0.05) when the salesperson discounts an important $(M=5.31)$ or a less-important attribute $(M=5.48)$ in the two-sided scenarios, contrary to H1. Figure 2 presents these results.

Trustworthiness mediation. To verify whether the path between remark condition and trustworthiness occurs by activating persuasion knowledge, we use a mediation analysis, based on Zhao et al. (2010) and Hayes (2013). In this analysis, the remark condition (dummy coded) was the independent variable (only positive information $=0$; two-sided information $=1$ ), the persuasion knowledge index was the mediator and the perceived salesperson trustworthiness was the dependent variable. The path between remark condition and the persuasion knowledge index was significant ( $a=-0.70$; $t=-21.66 ; p<0.001$ ), as was the path between mediator variable and perceived trustworthiness $(b=-0.94 ; t=-6.33 ; p<0.001)$. The indirect path was also significant $(a \times b=0.66 ; z=6.09 ; p<0.001)$ and does not present zero or the null effect in the confidence interval (95 per cent with 5,000 resamples), which ranges from 0.45 to 0.90 . Finally, the direct path is not significant $\left(c^{\prime}=0.21 ; t=1.46 ; p=0.14\right)$, revealing that the activation of persuasion knowledge fully mediates this effect. 

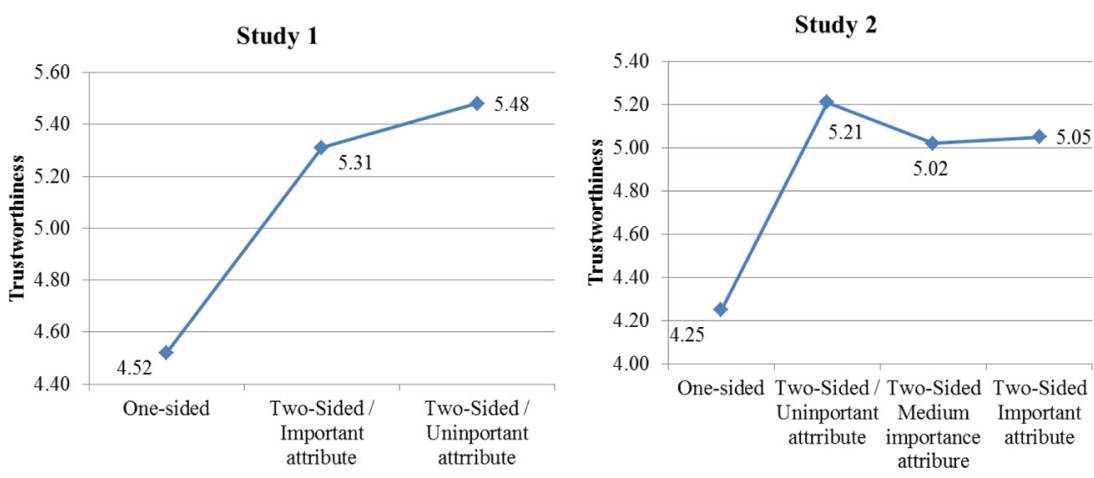

\section{Discounted attribute importance}

Study 3

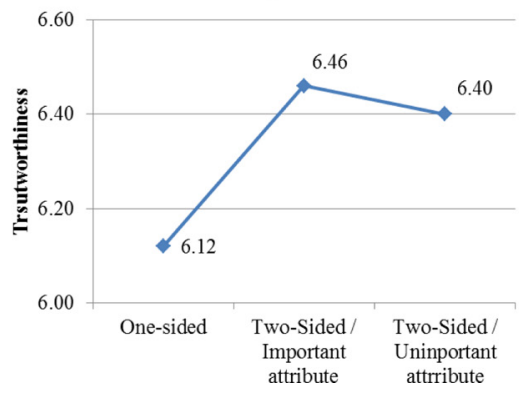

Figure 2.

The effect of two-sided communication and attribute importance on trustworthiness

Purchase intentions. Both communication manipulation $(F[1,468]=39.222, p<0.001)$ and attribute manipulation $(F[1,468]=25.410, p<0.001)$ yield a significant main effect on purchase intentions. Notably, there is a significant effect from the interaction between communication and attribute manipulations on purchase intentions $(F[1,468]=15.513, p<0.001)$.

As expected under $H 3$, when the discounting attribute is important, two-sided communication $(M=3.24)$ presents lower purchase intentions than one-sided communication $(M=4.35 ; F[1,250]=54.616, p<0.001)$. However, when the discounting attribute is considered to be unimportant (i.e. the need for ironing), two-sided communication presents a similar level of purchase intentions than one-sided communication $\left(M_{\text {one-sided }}=4.47\right.$ and $\left.M_{\text {two-sided }}=4.21 ; F[1,218]=2.595, p=0.10\right)$. These findings provide evidence that two-sided communication has no detrimental effect on purchase intentions when the attribute focus of the negative remark is of little importance to the subjects. Moreover, in planned comparisons, there is a significant effect of the attribute on purchase intentions when the participants were exposed to two-sided communication $(F[1,239]=34.226, p<0.001)$. In this condition, individuals exposed to the non-important attribute $(M=4.21)$ present higher purchase intentions than individuals exposed to the important attribute $(M=3.24)$. Additionally, participants in the one-sided condition exposed to an important attribute show no difference in terms of purchase intentions compared to participants exposed to an unimportant attribute $(F[1,229]=0.756, p=0.38)$. We present these findings supporting $H 3$ in Figure 3. 
$\mathrm{EJM}$

$50,9 / 10$

10

Purchase intentions mediation. To test H4, we used a mediation analysis and considered the message sidedness as the independent variable, trustworthiness as the mediator and purchase intentions as the dependent variable. First, we found that the message sidedness has a positive effect on trustworthiness $(a=0.88 ; t=8.07 ; p<$ $0.001)$. Trustworthiness has a positive effect on purchase intentions $(b=0.22 ; t=4.89$; $p<0.001)$. The indirect effect is positive $(a-b=0.20 ; z=4.19 ; p<0.001)$, and the 95 per cent bootstrap confidence interval $(0.10$ to 0.32$)$ for the indirect effect does not include zero. However, the direct path from message sidedness to purchase intentions is significant $(c=-0.90 ; t=-7.67 ; p<0.001)$. These results, providing support to $H 4$, indicate that trustworthiness partially mediates the effect of message sidedness on purchase intentions.

\section{Discussion}

The results from the first study partially support $H 1$ because a two-sided communication from a salesperson results in consumers perceiving higher trustworthiness compared with a one-sided communication. This phenomenon occurs through the use of persuasion knowledge because the path between communication and trustworthiness is fully mediated by the use of persuasion knowledge. However, contrary to $H 1$, the effect of the sidedness message happens regardless of the level of importance of the discounting attribute used in the two-sided communication.

Our evidence also supports the hypothesis that negative information from a salesperson decreases purchase intentions, providing evidence for the trade-off associated with the two-sided communication (i.e. it increases salesperson trustworthiness but decreases purchase intention). However, we found that this trade-off only occurs when discounting an important attribute in the two-sided communication. When the attribute is not important for individuals, their purchase intentions do not decrease.

In addition to the direct effect (and the conditioning effect from attribute importance), the purchase intention is also indirectly influenced by the message sidedness. We found that message sidedness increases trustworthiness that, in turn, positively increases purchase intentions.

However, it might be argued that the unimportant attribute selected for this study is a moderately important attribute and not particularly "unimportant" (the mean for the necessity of ironing was 3.30), which might be why trustworthiness was enhanced. To address this issue, we conducted a follow-up experiment with 161 respondents from

Figure 3.

The effect of two-sided communication and attribute importance on purchase intentions
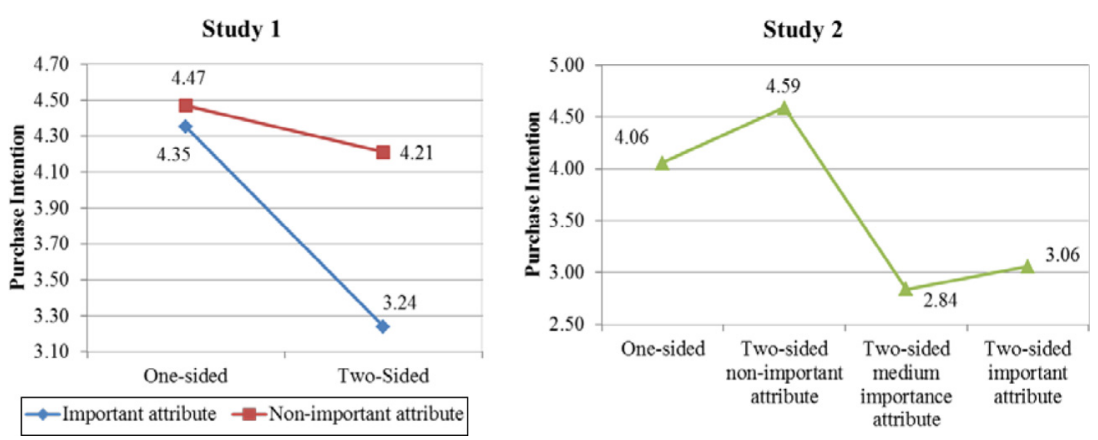
MTurk, where an even less important attribute was discounted - the thermal properties of the jeans ( $M=2.85$ in a scale of seven-points). Similar results for trustworthiness and purchase intentions were found.

Discounted attribute importance

\section{Study 2}

We designed this study to perform the following:

- test three levels of attribute importance;

- use a different product context to improve the external validity;

- use more attributes in the salesperson communication (five attributes), instead of only two as in the previous study;

- test the effects of two-sided communication on expertise; and, finally; and

- test if two-sided messages can also positively influence future behavioral intentions because the literature indicate that trust may have positive effects on intentions to return and recommendation in the future (Kim et al., 2009).

\section{Participants and design}

Participants were 221 undergraduate students (47.4 per cent male, $M_{\text {age }}=23.64, S D=$ 6.21) from a large university who voluntarily participated in an online survey. No screening procedure was used to remove participants from the analysis. This study follows a single-group (four conditions) between-subjects design with three levels of attribute importance in a two-sided message and a one-sided message condition. We use only one group for the one-sided message because we found no difference in Study 1 in the dependent variables because of attribute importance between the one-sided groups. We randomly assigned participants across treatments.

\section{Procedures and stimuli}

In this study, we used laptop computers as the product category. The attributes were chosen based on the results of a pretest. We used processor speed, audio quality, RAM and technical assistance as fixed attributes, and we varied battery life, USB entry, and packaging as the manipulation of very important, median important and non-important attributes, respectively. We also changed the relative position - first or last - of the negative message to test for order effects. Because we did not find any difference $(\phi>$ $0.10)$ based on the order of the negative comment, we pulled the data altogether. Table II presents the various scenarios.

After reading the scenario, respondents answered items related to the dependent variables.

\section{Measures}

Trustworthiness $(\alpha=0.91)$ and purchase intentions were measured by the same scale used in the first study. Persuasion knowledge was measured by an open question, and the two coders had an 88.3 per cent of agreement rate. Additionally, we measured salesperson perceived expertise using Ohanian (1990) $(\alpha=0.94)$ and consumer involvement with the product category as a covariate using a scale based on Ratchford (1987) $(\alpha=0.70)$. This variable had no influence on purchase intentions $(F[1,216]=$ $0.060, p=0.80)$. 
EJM

$50,9 / 10$

Table II.

Scenarios used in Study 2

\section{Communication manipulation}

Two-sided

\section{Attribute importance antation}

High This laptop has a high battery

importance life, a fast processor, good

audio quality, high ram

memory and has technical

Medium

assistance around the country
This laptop has a low battery life but has a fast processor, good audio quality, high ram memory and has technical assistance around the country

This laptop has only one USB entry but has a fast processor, good audio quality, high ram memory and has technical assistance around the country

Low importance

This laptop has poor packaging but has a fast processor, good audio quality, high ram memory and has technical assistance around the country

The two future behavioral intentions - intentions to return and to recommend - were measured by two items adapted from Zeithaml et al. (1996).

Results

Manipulations checks. Participants in the two-sided condition correctly indicated a higher mean for the "salesperson made a positive and negative remark about the laptop" question $(M=5.38)$ than participants in the one-sided condition $(M=2.33 ; F[1,210]=$ 41.38, $p<0.001)$.

Participants in the low importance attribute condition $(M=3.97)$ perceive the packaging as less important than participants in the medium importance condition $(M=5.64 ; F[1,100]=21.76, p<0.001)$. Likewise, participants in the USB entry condition (medium importance) perceive this attribute to be of lower importance than participants in the battery life condition $(M=6.22 ; F[1,109]=4.78, p<0.05)$, and there is a significant difference between low and high importance attributes $(F[1,105]=$ 50.71, $p<0.001$ ).

Trustworthiness. There is a main effect from message/attribute importance on perceived salesperson trustworthiness $(F[3,217]=5.11, p<0.005)$. When the consumer is exposed to a one-sided message, the $(M=4.25)$ perceived salesperson trustworthiness is lower than when the salesperson makes a two-sided communication with an unimportant attribute $(M=5.05)$, a medium importance attribute $(M=5.02)$ and a highly important attribute $(M=5.21)$. Again, contrary to $H 1$, there is no difference on perceived salesperson trustworthiness among the different attribute levels $(\phi>0.10)$. This result is presented in Figure 2.

Trustworthiness mediation. The path between the remark condition and the persuasion knowledge index was significant $(a=-0.18$; $t=-2.86$; $p<0.01)$, which was also true for the path between the mediator variable and perceived trustworthiness $(b=-0.84 ; t=-3.59 ; p<0.001)$. There is an indirect effect $(a \times b=0.15 ; z=2.24 ; p<$ $0.05)$, which ranges from 0.04 to 0.35 . However, the direct path is significant $\left(c^{\prime}=0.75\right.$; $t=3.39 ; p<0.001)$.

Purchase intentions. The message/attribute importance presents a main effect $(F[3,217]=13.54, p<0.001)$ on purchase intentions. By paired comparisons, we 
reinforce the previous findings because individuals exposed to the one-sided condition $(M=4.06)$ and individuals exposed to a two-sided condition with a non-important attribute discounted $(M=4.59)$ show no difference regarding purchase intentions $(F[1$, $108]=3.18, p=0.07$ ), whereas participants exposed to the one-sided condition present different purchase intentions from participants in two-sided conditions with medium importance $(M=2.84, p<0.001)$ and highly important discounted attributes $(M=3.06$, $p=0.001$ ). These results are presented in the Figure 3 .

Purchase intentions mediation. We found that the message sidedness has a positive effect on trustworthiness $(a=0.84 ; t=3.85 ; p<0.001)$, and trustworthiness has a positive effect on purchase intentions $(b=0.20 ; t=2.55 ; p<0.05)$. The indirect effect is positive $(a \times b=0.17 ; z=2.13 ; p<0.05)$, and the 95 per cent bootstrap confidence interval for the indirect effect does not include zero (0.04 to 0.39$)$. The direct path from message sidedness to purchase intentions is significant $(c=-0.77 ; t=-2.89 ; p<0.01)$. As in Study 1, these results support $H 4$.

Expertise. There is no effect of message sidedness/attribute importance on perceived salesperson expertise $(F[3,217]=0.59, p=0.62)$. Subjects exposed to one-sided $(M=$ 3.56), two-sided/non-important attribute $(M=3.81)$, two-sided/medium importance attribute $(M=3.80)$ and two-sided/highly important attribute $(M=3.90)$ conditions presented similar perceptions about salesperson expertise.

Behavioral intentions. Additionally, to provide evidence that a two-sided message may increase other positive behavioral intentions (from the retailer's perspective), we ran two mediation analyses to show that the communication condition has an effect on the respondents' intentions to "recommend the store" and "return to the store", as mediated by perceived salesperson trustworthiness.

First, with the intention to recommend the store as the dependent variable, the model shows that communication has a significant effect on trustworthiness $(a=0.79 ; t=2.85$; $p<0.01$ ) and that trustworthiness has a significant effect on the intention to recommend the store $(b=0.44 ; t=4.55 ; p<0.001)$. The indirect effect is positive $(a \times b=0.35 ; z=$ $2.43 ; p<0.05)$ and ranged from 0.09 to 0.75 , and there is no direct effect $\left(c^{\prime}=-0.24\right.$; $t=-0.84 ; p=0.40$ ).

Likewise, when the return to the store intention was the dependent variable, the path between trustworthiness and return intention was positive $(b=0.39 ; t=4.48 ; p<0.001)$, which was similar for the indirect path from communication to return intention $(a \times b=$ $0.31 ; z=2.42 ; p<0.05)$. There is no direct effect $\left(c^{\prime}=-0.12 ; t=-0.45 ; p=0.64\right)$.

\section{Discussion}

The findings of Study 2 extended the findings of the Study 1 by showing that a two-sided message discounting medium or high importance attributes trigger similar behaviors in term of consumer willingness to purchase. Both of these conditions reduce consumer intentions to buy the product, which is different from that which occurs when a non-important attribute is discounted by the salesperson (i.e. there are similar purchase intentions regarding the one-sided message).

We qualified the previous results by:

- replicating the same findings with a different quantity of the attributes communicated by the salesperson (five instead of two); 
EJM

$50,9 / 10$

14

- testing the results in a different product context and with a different sample; and

- evidencing the communication's indirect effect on intentions to recommend the store and to return to the store.

In addition, this study allowed us to verify that there is no impact of message sidedness on expertise. These findings reinforce our assumption that in a persuasion context such as a retailing, a salesperson's two-sided communication has a relevant effect on perceived trustworthiness but not on the perceived expertise facet of credibility.

\section{Study 3: field experiment}

To add external validity to our research, we sought to replicate the findings of Studies 1 and 2 in a field setting with actual purchase decisions. We also aimed to increase the generality of the findings by changing the target product and, as a consequence, the discounting attributes.

\section{Participants and design}

Participants were 128 students (73.4 per cent female, $M_{\text {age }}=22.89, S D=7.37$ ). No screening procedure was used to remove participants from the analysis.

The design was the same of the first study: a 2(communication conditions: one-sided/ two-sided) $\times 2$ (attribute conditions: important/non-important) between-subjects.

\section{Procedures and stimuli}

A retail booth of chocolate truffles was set up in the main entrance of a university campus. The retail outlet was operated by the experimenter, who acted as a salesperson. The communication manipulation was administered by the experimenter, who initially addressed all the potential consumers by saying, "Hi. We are doing a test market for this product. Feel free to check out our truffles". The manipulation was executed by the experimenter after the consumer approached the booth and included one of the four conditions presented in Table III.

The chocolate truffles' attributes were chosen based on a previous pre-test $(n=42)$, which demonstrated that taste $(M=6.62)$ and not being "sickening-sweet" $(M=6.43)$ were the most important attributes in chocolate truffles. However, packaging was not so important $(M=4.90)$ and was perceived as less important than both taste $(t=5.29 ; p<$ $0.001)$ and not being sickening-sweet $(t=4.81 ; p<0.001)$.

After this encounter, the consumer decided whether to buy (or not buy) the product. If the consumer decided to buy, the truffles were sold for US\$1.00 each. Independently of the consumer's decision, after he or she left the store, another experimenter

Table III.

Scenarios used in Unimportant Study 3

\section{Communication}

One-sided

Two-sided

\section{Attribute importance}

Important

These truffles are tasty and not sickening-sweet

Unimportant These truffles are tasty and the packaging is resistant
These truffles are tasty, but somewhat sickening-sweet These truffles are tasty, but the packaging is not resistant 
approached and invited the consumer to answer a quick survey containing the measures used in the study. The experiment was run for five days to minimize the chances that students would be able to discuss the experimental procedures among themselves. Finally, none of the experimenters reported knowing any of the participants.

\section{Measures}

We measured trustworthiness $(\alpha=0.91)$ through the same scale used in the previous studies. The purchase behavior (not intentions, as in the previous studies) was coded as a binary answer $(0=$ not buy $/ 1=$ buy $)$.

Whether the consumer was with other consumers (63.3 per cent were together with other consumers) had no influence on perceived trustworthiness $(F[1,123]=0.01 ; p=$ 0.92 ) or purchase behavior (Wald $\chi^{2}(1)=0.10 ; p=0.75$ ).

\section{Results}

Manipulations checks. When the participants were exposed to the two-sided condition, they correctly indicated a higher mean for the "salesperson made positive and negative remark about the truffles" question $(M=6.07)$ than participants in the one-sided condition $(M=4.94 ; F[1,124]=40.57 ; p<0.001)$. There is no effect indicated for attribute manipulation $(F[1,124]=2.40 ; p=0.12)$ or interaction $(F[1,124]=0.00 ; p=$ $0.93)$ in the communication manipulation check.

In the attribute manipulation, taste $(M=6.60)$ and not being sickening-sweet $(M=$ 6.05) are attributes that are significantly more important than packaging $(M=4.16$; $t=$ 12.71 and $t=10.24 ; \mathrm{df}=127 ; p<.001)$. There are no effects related to communication manipulation or interaction on the attribute manipulation check $(p>0.05)$.

Trustworthiness. Neither attribute manipulation $(F[1,123]=0.02, p=0.88)$ nor interaction $(F[1,123]=0.05, p=0.81)$ present significant effects on perceived salesperson trustworthiness. However, communication manipulation presents a significant main effect $(F[1,123]=4.46, p<0.05)$. The findings are similar to those from previous studies. Participants exposed to a one-sided condition $(M=6.12)$ presented a lower perception of salesperson trustworthiness than subjects in the two-sided condition $(M=6.43)$. There is no difference between two-sided communication with an important attribute $(M=6.46)$ and two-sided communication with a less-important attribute $(M=$ $6.40 ; F[1,62]=0.50 ; p=0.82$ ). These results are presented in the Figure 2 .

Purchase. A binary logistic regression demonstrated the effects of the interaction between communication sidedness and attribute (Wald $\chi^{2}(1)=4.19 ; p<0.05$ ) on purchase behavior. In the important attribute condition, participants exposed to the one-sided condition (66.7 per cent) purchased the product more than participants exposed to the two-sided communication (34.4 per cent; $\chi^{2}(1)=6.77 ; p<0.01$ ). However, in the non-important attribute condition, participants exposed to the one-sided (56.7 per cent) and two-sided (60.6 per cent) conditions present similar purchase behavior $\left(\chi^{2}(1)=\right.$ $0.10 ; p=0.75)$. These results reinforce $H 3$.

Additionally, in the two-sided condition, participants bought more when the attribute was unimportant (60.6 per cent) than when the attribute was important (34.4 per cent; $\left.\chi^{2}(1)=4.48 ; p<0.05\right)$. There is no difference in the purchase between participants exposed to the important (66.7 per cent) and unimportant attributes (56.7 per cent; $\chi^{2}(1)=0.66 ; p=0.41$ ) in the one-sided condition. These findings are

Discounted attribute importance

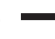


EJM

$50,9 / 10$

\section{6}

Figure 4.

Study 3: the effect of two-sided communication and attribute importance on purchase behavior

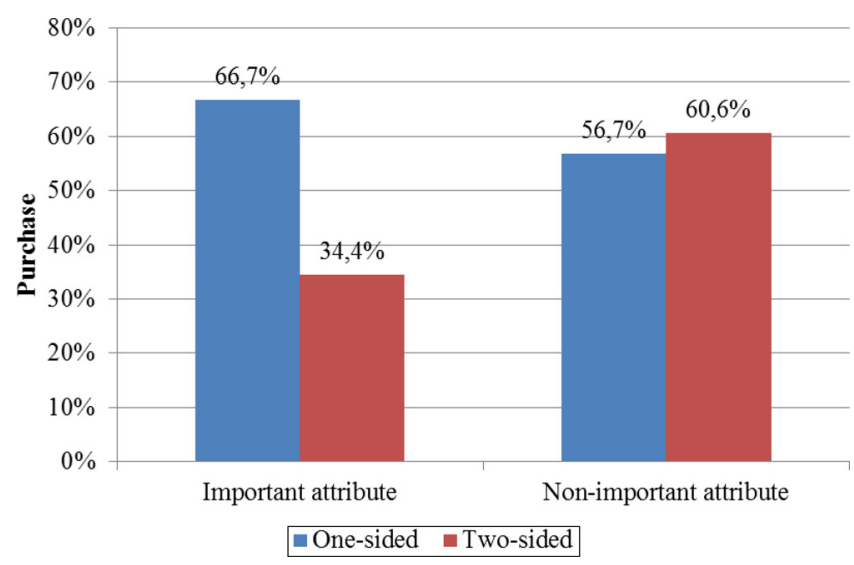

Discussion

The field experiment provides evidence that the phenomenon detected in our laboratory studies also occurs in real situations, which increases the external validity of our findings. In this study, using a behavioral measure as the dependent variable, we demonstrate that the trade-off between trustworthiness and purchase only occurs in a retail setting when the two-sided message discounts an important attribute.

\section{General discussion}

In essence, the previous literature highlights the trade-off associated with two-sided appeals, i.e. two-sided messages trigger more trustworthiness than one-sided messages, but the "price" paid for these gains is a lower probability of purchase by consumers because of the presentation of negative information about the product. Therefore, the question that guided this research was the following:

\section{Q3. Is this trade-off unavoidable?}

Our study answers this question with a "no". It is possible to enhance trustworthiness through a two-sided message without mitigating the intentions of buying by controlling for the importance of the negative information provided.

Across three experimental studies (two lab experiments and one field experiment) with different populations (students and nonstudents) and different products (jeans, laptops and chocolate truffles), the present research shows that a negative salesperson remark in a retail setting during an interaction with a consumer reduces the use of persuasion knowledge by the consumer and - as a result - enhances the consumer's perception of the salesperson's trustworthiness. This enhancement occurs through the elicitation of correspondent attribution (Jones and Davis, 1965) that makes the ulterior motives of the sales agent less apparent.

The positive effect of a two-sided message on a salesperson's trustworthiness remains similar across diverse levels of importance of the discounting attribute, which means that this effect is not moderated by the importance of the disclaimed message. One explanation for our result is that the act of discounting alone, rather than what specifically is discounted, may be the major cause of the trustworthiness gain, and greater than minimal discounting may not result in substantial trustworthiness gains 
(Stayman et al., 1987). Thus, it seems that a salesperson who acts contrary to normative behavior by making any negative comment (even one related to a non-important attribute of the product) makes his/her true disposition more evident.

Furthermore, a negative remark from a salesperson during an interaction with a consumer decreases the probability of purchase but only when an important attribute is disclaimed. The logic behind the moderating role of the importance of the discounting attribute is based on the influence that the communication has on the consumer's attitude. When an important attribute is disclaimed, the consumer has a lowered evaluation of these attributes than when an unimportant attribute is disclaimed. However, one may question why discounting an unimportant attribute does not reduce, at least a little, the willingness to purchase, compared to a one-sided message. We believe the trustworthiness gained with the two-sided message seems to play a pivotal role in relation to the loss of purchase intentions. Because in any case (discounting an important or unimportant attribute), a two-sided message enhances trustworthiness, and trustworthiness leads to higher buying intentions, as our findings show; when a salesperson uses a two-sided communication to discount a non-important attribute of the product, gains in trustworthiness seem to compensate for losses in the evaluation of the product. Hence, purchase intentions do not significantly weaken, compared to a one-sided message. Conversely, when an important attribute is discounted, the loss in the evaluation of product attributes is higher than the gains from perceived trustworthiness.

It might be argued that our findings reveal that two-sided messages are no more effective than a one-sided message because purchase intentions are not enhanced by including a negative attribute in the communication. However, if the two-sided message is not more effective, it is also not less effective: purchase intentions are not decreased when an unimportant attribute is disclaimed in a two-sided message compared with a one-sided message. Moreover, the inclusion of a negative remark from a salesperson leads to stronger consumer intentions to return to that store in the future and to recommend the store to family and friends because of enhanced trustworthiness. Therefore, the use of two-sided messages (discounting an attribute with low importance) does not bring any harm for the company and does lead to positive consequences.

\section{Theoretical and managerial contributions}

Although two-sided messages might be easily used by salespeople during interactions with a consumer in a retail setting, the literature is surprisingly sparse (Hunt and Smith, 1987 is an exception). From a theoretical perspective, the present paper makes two main contributions. First, we tested and empirically found that the importance of the discounting attribute does not moderate the effect of a salesperson's two-sided messages on a consumer's trustworthiness but can reduce the negative effect on purchase intentions in retail settings. Previous research on two-sided messages, following the prescriptions of inoculation theory (McGuire, 1964), has simply disclaimed unimportant attributes in their studies to mitigate consumers' lower purchase intentions resulting from the presentation of negative information about the product. However, surprisingly little research has focused on the importance of the disclaimed attribute and its impact on advertising effectiveness.

Second, our research shows that the trade-off phenomenon is avoidable because two different underlying mechanisms account for the impact of two-sided communication on
Discounted attribute importance 
EJM

$50,9 / 10$

18

trustworthiness and purchase intentions. On trustworthiness, negative information (regardless the level of importance), unexpected by the consumer, leads to lower activation of consumer's persuasion knowledge and then to higher levels of trustworthiness (Campbell and Kirmani, 2000). However, presentation of only positive information by the salesperson leads to higher use of persuasion knowledge by the consumer. Essentially, the activation of persuasion knowledge explains how two-sided communication from a salesperson influences a consumer's perception of trustworthiness.

The weak effect on perceptions of salesperson expertise - the other facet of credibility beyond trustworthiness (Ohanian, 1990) - seems to reinforce the importance of the salesperson's willingness to tell the truth in persuasion settings, such as the retail and advertising contexts.

Regarding purchase intentions, we show that the level of purchase intentions reduced by discounting an important attribute is higher than is reduced by discounting an unimportant attribute. However, contrary to some literature that favored the two-sided treatments (Etgar and Goodwin, 1982; Golden and Alpert, 1987; Kamins, 1989; Kamins et al., 1989), we have found no situation where a two-sided message from a salesperson leads to more purchase intentions than a one-sided message. Different research contexts (i.e. sales vs advertising and use of celebrities) seem to have a role in these results because Hunt and Smith (1997) - the only authors to study a two-sided message in selling appeals - found that participants exposed to the one-sided message were more likely to consider the product for purchase than those exposed to the two-sided message.

Moreover, we found that trustworthiness is the path between two-sided messages and positive consumer behavioral intentions that are in addition to purchase intentions. By enhancing the trustworthiness of the sales representative, the intentions to visit the store again and to recommend the store tend to increase as well. These are desirable consequences of salesperson trustworthiness. Thus, this strategy seems to align with the notion of relationship marketing, which emphasizes building relationships rather than making short-term sales (Sheth and Parvatiyar, 1995). In fact, the relationship marketing literature shows that enhanced trust in salespersons has a long-term effect, including helping build stronger and more profitable relationships with consumers (Doney and Cannon, 1997; Swan et al., 1999).

From a managerial point of view, our findings show that retailers can benefit by encouraging frontline employees to use negative feedback along with positive feedback as part of their communication strategy. A negative comment from a salesperson can have positive effects for the sales agent and for the store. The use of two-sided communication increases the levels of sincerity and honesty that the consumer perceives in the sales agent. Consequently, consumers reveal more positive behavioral intentions, such as intentions to purchase, and the willingness to return to the store and to recommend the store to another person.

However, it is important to make note of the following condition in this regard: the importance of the disclaimed attribute. As shown herein, a negative remark related to an important attribute allows a trade-off to occur between trustworthiness and purchase intentions, whereas a negative remark related to an unimportant attribute will eliminate this trade-off, i.e. trustworthiness will be increased, but the willingness to buy will not be decreased. Therefore, for the appropriate use of two-sided appeals, retailers should first 
identify which attributes are considered more and less important from the consumers' perspective and choose one that is unimportant for discounting in two-sided messages. An ordinary survey with a group of consumers might yield this basic information.

Note that we do not indicate that sales clerks should lie to consumers to improve their sincerity ratings. Instead, we suggest that they may express their honest thoughts (positive and negative) about products more freely.

Notably, our recommendation is restricted to unfamiliar consumers, i.e. consumers who do not have a previous relationship with the sales agent. When there is an ongoing relationship between a consumer and an employee, the effect of a two-sided message is likely to be reduced because the level of trustworthiness may already be high.

\section{Limitations and suggestions for future studies}

It is worth to acknowledge that in the field study, although our attempt to have impartial experimenters, they could have created part of the effect found by not being blind and changing their behavior (even if without intent). Besides, as in most two-sided studies, we used an unknown store/product brand and salesperson, so no prior attitude may be assumed. However, we recognize that the effectiveness of a persuasive message is likely to be influenced by the receiver's prior attitude toward a product and by his/her prior relationship with the salesperson. Therefore, future research could examine the phenomenon in other consumer-employee situations and in situations in which there is a prior attitude (e.g. when the selling appeal is for a familiar or known brand). In this case, researchers might explore the potentially diverse effects of two-sided messages that depend on whether this prior attitude is favorable (or not). For instance, Crowley and Hoyer (1994) propose that two-sided messages are more effective than one-sided messages in changing negative attitudes because a message containing negative information may be more likely to fall into the receiver's "latitude of acceptance" and encounter less resistance as a result.

We attempted to control for other message characteristics, such as the correlation between negative and positive attributes (by keeping it not high) and the order of presentation (by counterbalancing the scenarios, positive attribute following negative attribute versus negative attribute following positive attribute). However, several unanswered questions remain regarding the optimal structure of two-sided messages, such as how these message characteristics interact with one another. For example, future research could explore the amount of information - and the importance of the information - to check the impact of this importance across the different amounts of information (positive and negative) presented. In addition, despite not measuring the level of processing effort in our experiments because it was an ordinary retail exchange we assumed that our respondents were operating with low processing activity. Hence, future work could explore the trade-off phenomenon by taking into account the moderating effect of different processing-effort conditions.

Another path of research could be to explore situations where two-sided messages could lead to higher purchase intentions (compared to one-sided messages). One possible situation is when the consumers already have a negative attitude toward the product or brand. As we said above, a two-sided message tends to be more effective on consumers that are initially unfavorable to the brand because in being more consistent with the receiver's attitudinal schema, it may encounter less resistance (Crowley and Hoyer, 1994; Eisend, 2006).

\section{Discounted attribute importance}

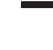


$\mathrm{EJM}$

$50,9 / 10$

\section{References}

Ahluwalia, R. (2000), "Examination of psychological processes underlying resistance to persuasion”, Journal of Consumer Research, Vol. 27 No. 2, pp. 217-232.

Basso, K., Santos, C.P. and Gonçalves, M.A. (2014), "The impact of flattery: the role of negative remarks", Journal of Retailing and Consumer Services, Vol. 21 No. 2, pp. 185-191.

Bushman, B.J. and Bonacci, A.M. (2002), "Violence and sex impair memory for television ads", Journal of Applied Psychology, Vol. 87 No. 3, pp. 557-564.

Campbell, M.C. and Kirmani, A. (2000), "Consumers' use of persuasion knowledge: the effects of accessibility and cognitive capacity on perceptions of an influence agent", Journal of Consumer Research, Vol. 27 No. 1, pp. 69-83.

Cornelis, E., Cauberghe, V. and De Pelesmacker, P. (2014), "Regulatory congruence effects in two-sided advertising: the mediating role of processing fluency and processing depth", European Journal of Marketing, Vol. 48 Nos 7/8, pp. 1451-1465.

Crowley, A.E. and Hoyer, W.D. (1994), "An integrative framework for understanding two-sided persuasion”, Journal of Consumer Research, Vol. 20 No. 4, pp. 561-574.

Doney, P.M. and Cannon, J.P. (1997), "An examination of the nature of trust in buyer-seller relationships”, Journal of Marketing, Vol. 61 No. 2, pp. 35-51.

Drachman, D., DeCarufel, A. and Insko, C.A. (1978), "The extra credit effect in interpersonal attraction", Journal of Experimental Social Psychology, Vol. 14 No. 5, pp. 458-465.

Eagly, A.H. and Chaiken, S. (1993), The Psychology of Attitudes, Harcourt Brace Jovanovich College Publishers, Orlando.

Ein-Gar, D., Shiv, B. and Tormala, Z.L. (2012), "When blemishing leads to blossoming: the positive effect of negative information", Journal of Consumer Research, Vol. 38 No. 5 , pp. 846-859.

Eisend, M. (2006), “Two-sided advertising: a meta-analysis”, International Journal of Research in Marketing, Vol. 23 No. 2, pp. 187-198.

Eisend, M. (2007), "Understanding two-sided persuasion: an empirical assessment of theoretical approaches", Psychology and Marketing, Vol. 24 No. 7, pp. 615-640.

Eisend, M. (2010), "Explaining the joint effect of source credibility and negativity of information in two-sided messages", Psychology and Marketing, Vol. 27 No. 11, pp. 1032-1049.

Etgar, M. and Goodwin, S.A. (1982), "One-sided versus two-sided comparative message appeals for new brand introductions", Journal of Consumer Research, Vol. 9, pp. 460-465.

Friestad, M. and Wright, P. (1994), "The persuasion knowledge model: how people cope with persuasion attempts", Journal of Consumer Research, Vol. 21 No. 1, pp. 1-31.

Friestad, M. and Wright, P. (1999), "Everyday persuasion knowledge”, Psychology and Marketing, Vol. 16 No. 2, pp. 185-194.

Golden, L.L. and Alpert, M.I. (1987), "Comparative analyses of the relative effectiveness of one-sided and two-sided communications for contrasting products", Journal of Advertising, Vol. 16 No. 1, pp. 18-28.

Hastak, M. and Park, J. (1990), "Mediators of message sidedness effects on cognitive structure for involved and uninvolved audiences", in Goldberg, M.E., Gorn, G. and Pollay, R.W. (Eds), Advances in Consumer Research, Association for Consumer Research, Provo, UT, pp. 329-336.

Hayes, A.F. (2013), Introduction to Mediation, Moderation, and Conditional Process Analysis, The Guilford Press, New York, NY. 
Hunt, J.M. and Smith, M.F. (1987), “The persuasive impact of two-sided selling appeals for an unknown brand name", Journal of the Academy of Marketing Science, Vol. 15 No. 1, pp. 11-18.

Jones, E.E. and Davis, K.E. (1965), "From acts to dispositions: the attribution process in person perception", in Berkowitz, L. (Ed.), Advances in Experimental Social Psychology, Vol. 2, Academic Press, New York, NY, pp. 219-266.

Kamins, M.A. (1989), “Celebrity and non celebrity advertising in a two sided context”, Journal of Advertising Research, Vol. 29 No. 3, pp. 34-42.

Kamins, M.A. and Assael, H. (1987), "Two-sided versus one-sided appeals: a cognitive perspective on argumentation, source derogation, and the effect of disconfirming trial on belief change", Journal of Marketing Research, Vol. 24 No. 1, pp. 29-39.

Kamins, M.A., Brand, M.J., Heoke, S.A. and Moe, J.C. (1989), "Two-sided versus one-sided celebrity endorsements: the impact on advertising effectiveness and credibility", Journal of Advertising, Vol. 18 No. 2, pp. 4-10.

Kamins, M.A. and Marks, L.J. (1987), "Advertising puffery: the impact of using two-sided claims on product attitude and purchase intention”, Journal of Advertising, Vol. 16 No. 4, pp. 6-15.

Kim, T.T., Kim, W.G. and Kim, H.B. (2009), "The effects of perceived justice on recovery satisfaction, trust, word-of-mouth, and revisit intention in upscale hotels", Tourism Management, Vol. 30 No. 1, pp. 51-62.

McGuire, W.J. (1964), "Inducing resistance to persuasion”, in Berkowitz, L. (Ed.), Advances in Experimental Social Psychology, Vol. 1, McGraw-Hill, New York, NY, pp. 192-229.

Main, K.J., Dahl, D.W. and Darke, P.R. (2007), "Deliberative and automatic bases of suspicion: empirical evidence of the sinister attribution error", Journal of Consumer Psychology, Vol. 17 No. 1, pp. 59-69.

Ohanian, R. (1990), "Construction and validation of a scale to measure celebrity endorsers' perceived expertise, trustworthiness, and attractiveness", Journal of Advertising, Vol. 19 No. 3, pp. 39-52.

Okechuku, C., Frost, M. and Porchuk, M. (1993), "The moderating effect of disclaimer importance on the effectiveness of two-sided advertising", in Raaij, W.F.V. and J. Bamossy, G.J. (Eds), European Advances in Consumer Research, Vol. 1, Association for Consumer Research, UT, pp. 168-175.

Pechmann, C. (1992), "Predicting when two-sided ads will be more effective than one-sided ads: the role of correlational and correspondent inferences”, Journal of Marketing Research, Vol. 29, pp. $441-453$.

Ratchford, B.T. (1987), "New insights about the FCB grid", Journal of Advertising Research, Vol. 27 No. 4, pp. 24-38.

Sawyer, A.G. (1973), "The effects of repetition of refutational and supportive advertising appeals", Journal of Marketing Research, Vol. 10 No. 1, pp. 23-33.

Schlosser, A.E. (2011), "Can including pros and cons increase the helpfulness and persuasiveness of online reviews? The interactive effects of ratings and arguments", Journal of Consumer Psychology, Vol. 21 No. 3, pp. 226-239.

Settle, R.B. and Golden, L.L. (1974), "Attribution theory and advertiser credibility”, Journal of Marketing Research, Vol. 11 No. 2, pp. 181-185.

Sheth, J.N. and Parvatiyar, A. (1995), "The evolution of relationship marketing", International Business Review, Vol. 4 No. 4, pp. 397-418.

Shu, S.B. and Carlson, K.A. (2014), "When three charms but four alarms: identifying the optimal number of claims in persuasion settings", Journal of Marketing, Vol. 78 No. 1, pp. 127-139. 
$\mathrm{EJM}$

$50,9 / 10$

Smith, R.E. and Hunt, S.D. (1978), "Attributional processes and effects in promotional situations”, Journal of Consumer Research, Vol. 5 No. 3, pp. 149-158.

Stayman, D., Hoyer, W. and Leon, R. (1987), "Attribute importance in discounting product features in advertising” in Douglas, S.P. et al. (Eds), AMA Educators' Proceedings, Toronto, No. 53.

Swan, J.E., Bowers, M.R. and Richardson, L.D. (1999), "Customer trust in the salesperson: an integrative review and meta-analysis of the empirical literature", Journal of Business Research, Vol. 44 No. 2, pp. 93-107.

Swinyard, W.R. (1981), "The interaction between comparative advertising and copy claim variation”, Journal of Marketing Research, Vol. 18 No. 2, pp. 175-186.

Zeithaml, V.A., Berry, L.L., Parasuraman, A. (1996), "The behavioral consequences of service quality", Journal of Marketing, Vol. 60 No. 2, pp. 31-46.

Zhao, X., Lynch, J.G. and Chen, Q. (2010), "Reconsidering Baron and Kenny: myths and truths about mediation analysis", Journal of Consumer Research, Vol. 37 No. 2, pp. 197-206.

\section{Further reading}

Smith, R.E. and Swinyard, W.R. (1983), "Attitude-behavior consistency: the impact of product trial versus advertising”, Journal of Marketing Research, Vol. 20 No. 3, pp. 257-267. 


\begin{tabular}{lccccccc}
\hline & \multicolumn{2}{c}{ Study 1 } & \multicolumn{2}{c}{ Study 2 } & \multicolumn{2}{c}{ Study 3 } \\
Measure/Items & Mean (SD) & $\alpha$ & Mean (SD) & $\alpha$ & Mean (SD) & $\alpha$ \\
\hline Trustworthiness & $4.93(1.22)$ & 0.93 & $4.86(1.49)$ & 0.91 & $6.28(0.93)$ & 0.91 \\
$\quad$ Sincere & $4.98(1.24)$ & & $5.09(1.52)$ & & $6.23(1.05)$ & \\
Honest & $5.05(1.29)$ & & $4.95(1.62)$ & & $6.39(0.94)$ & \\
Trustworthy & $4.78(1.37)$ & & $4.55(1.74)$ & & $6.22(1.03)$ &
\end{tabular}

Perception of unexpected behavior

How do you consider the situation described, particularly the salesperson's behavior

$(1=$ unexpected $/ 7=$ expected $)$

$\begin{array}{cc}\text { One-sided } & \text { One-sided } \\ 5.29(1.13) & 5.45(1.47) \\ \text { Two-sided } & \text { Two-sided } \\ 4.07(1.66) & 4.38(1.67)\end{array}$

Purchase intention

How likely would you be to purchase this jacket/jeans $(1=$ very unlikely $/ 7=$ very likely)

$3.95(1.14) \quad 3.63(1.76)$

\section{Attributes importance}

Important-fit/battery life (one-sided)/taste Important-comfort/battery life (two-sided)/ not sickening

Medium-USB entry

Unimportant-needs

ironing/package/package

$\begin{array}{ccc}6.10(0.97) & 6.42(1.07) & 6.60(0.73) \\ 6.18(0.95) & 6.22(1.21) & 6.05(1.30) \\ & 5.64(1.58) & \\ 3.30(1.78) & 3.97(2.00) & 4.16(1.97) \\ & & \\ 0.47 & - & 0.45 \\ 0.03 & - & 0.20\end{array}$

Correlation between the attributes

$\begin{array}{llll}\text { (Important vs important) } & 0.47 & - & 0.45\end{array}$

(Important vs unimportant)

\section{Corresponding author}

Cristiane Pizzutti can be contacted at: crispizzutti@gmail.com

For instructions on how to order reprints of this article, please visit our website:

www.emeraldgrouppublishing.com/licensing/reprints.htm

Or contact us for further details: permissions@emeraldinsight.com 


\section{AUTHOR QUERIES}

\section{AUTHOR PLEASE ANSWER ALL QUERIES}

AQau-Please confirm the given-names and surnames are identified properly by the colours. $=$ Given-Name, $\mathbf{a}=$ Surname The colours are for proofing purposes only. The colours will not appear online or in print.

AQ1 - Please note that the following citations are not listed in the reference list. Please provide full details for these citations: Hovland et al. (1953); McGinnies and Ward (1980); Sirdeshmuck et al. (2002); DeCarlo (2005); Hunt and Smith (1997).

AQ2 - Please provide first column head for Tables I, II, and III. 